\section{Pertes de bien-être et pouvoir de marché dans l'agro-alimentaire français}

\author{
Pascal Lavergne $^{(*)}$ \\ Vincent Réquillart ${ }^{(*)}$
}

Michel Simioni $^{(*)}$
À la suite du travail de Harberger (1954), une importante littérature a été consacrée à l'analyse des pertes de bien-être résultant du pouvoir de marché. La mesure des pertes de bien-être peut s'appliquer non seulement à la définition des politiques optimales mais aussi à d'autres domaines. Par exemple, l'analyse antitrust nécessite de mesurer les effets sur le bien-être des consommateurs de la stratégie des firmes. La réglementation des fusions repose sur les implications en terme de bien-être des structures alternatives d'une industrie (voir Willig, 1991). L'industrie agro-alimentaire est particulièrement concernée par ces réglementations. Ainsi, le rachat de Perrier par Nestlé ne fut autorisé que sous des conditions strictes. De plus, selon une étude de la DG II (voir Jacquemin, Buigues et Ilzkovitz, 1989), d'éventuelles fusions horizontales pourraient entraîner une diminution importante de la concurrence dans cinq secteurs de l'industrie agro-alimentaire : fabrication de pâtes alimentaires, chocolaterie et confiserie, production d'apéritifs à base de vin, eaux minérales et boissons rafraîchissantes, bière et industrie du malt.

La mesure des pertes de bien-être résultant du pouvoir de marché est donc utile à la définition des politiques publiques. Cependant il n'existe pas de consensus quant à l'ordre de grandeur de ces pertes dans l'industrie manufacturière. En effet, les études empiriques fournissent un large éventail d'estimations. Certaines études concluent à des pertes faibles de l'ordre de $0,3 \%$ du PNB (voir Harberger, 1954, ou Gisser, 1989) alors que d' autres estiment des pertes pouvant représenter jusqu'à $7 \%$ du PNB (voir Jenny et Weber, 1983, ou Willner, 1983). Ces différences proviennent en partie des hypothèses faites sur les courbes de demande. Ainsi, les études les plus anciennes se bornent à l'utilisation d'une demande linéaire et fixent généralement la valeur de l'élasticité prix à un niveau donné. Les travaux les plus récents utilisent des demandes isoélastiques et étudient la sensibilité des résultats à une variation de l'élasticité prix de la demande. Enfin, un certain nombre de ces travaux utilisent des estimations des élasticités provenant d'autres études.

Une deuxième source de divergence entre les études concerne la façon dont les distorsions de prix sont évaluées. Dans la tradition de Bain, les distorsions de prix peuvent être estimées soit en utilisant les données sur la profitabilité des industries (voir, entre autres, Harberger, 1954, et Parker et Connor, 1979) soit la différence entre le prix des marques nationales et celui des marques de distributeurs (voir Connor et Peterson, 1992). Alternativement, des règles explicites de tarification peuvent être déduites de la théorie de l'oligopole. Ainsi, dans un jeu en quantité avec variations conjecturales, la marge relative prix-coût peut être exprimée comme une fonction croissante de l'indice de concentration de Herfindahl et Hirschman et comme une fonction 
décroissante de la valeur absolue de l'élasticité prix de la demande (voir Cowling et Waterson, 1976). Certains auteurs proposent une analyse à partir d'autres modèles de comportement tels que les modèles de firme dominante (voir Gisser, 1986, et Willner, 1989). Connor et Peterson (1994 et 1995) comparent ces différentes méthodes pour évaluer les pertes de bien-être dans l'agro-alimentaire américain. Selon ces auteurs, la méthode d'évaluation a un rôle sur le niveau absolu de pertes de bien-être mais n'influe que très peu sur le niveau relatif de ces pertes, les secteurs qui enregistrent les pertes les plus fortes sont les mêmes quelles que soient les méthodes. Les résultats obtenus dans notre article ne confirment pas cette conclusion.

Un point commun entre les différentes études empiriques est l'utilisation du concept de surplus marshallien, même si les auteurs mettent en avant ses limites. En effet, le surplus marshallien n'est pas, en général, une mesure exacte de la variation de surplus puisque l'utilité des consommateurs n'est pas maintenue constante le long de la courbe de demande. La mesure de la variation de surplus doit être basée sur l'utilisation de la courbe de demande hicksienne, ou demande compensée. Comme Willig (1976) l'a montré, la différence entre ces deux mesures est bornée et dans la plupart des cas inférieure aux erreurs liées à l'estimation de la demande. Cependant, cet argument n'est pas recevable dès lors que l'on s'intéresse aux pertes de bien-être. Même si les mesures marshallienne et hicksienne de la variation de surplus des consommateurs associées à une variation de prix sont voisines, il n'en est pas toujours de même des pertes de bien-être puisque ces dernières ne constituent qu'une fraction de la variation de surplus.

Le principal objectif de cet article est de mettre en évidence certaines difficultés pratiques rencontrées lorsque l'on désire évaluer des pertes de bien-être liées au pouvoir de marché. Comme précisé ci-dessus, ces difficultés sont reliées à la spécification de la courbe de demande, à la mesure de la marge prix-coût et au calcul des pertes hicksiennes de bien-être. Pour répondre à cet objectif, nous utilisons des données concernant l'agro- alimentaire français. Dans un premier temps, nous estimons les courbes de demande alimentaire en utilisant des données agrégées de consommation. Une importance particulière est alors donnée aux contraintes imposées par la théorie économique du consommateur. Parallèlement, les écarts prix-coûts marginaux sont mesurées en utilisant deux méthodes d'approximation. La première utilise des données de profitabilité des secteurs et la seconde repose sur l'utilisation d'une règle explicite de comportement. Ces résultats nous permettent de calculer les mesures marshallienne et hicksienne des pertes de bien-être. Le surplus hicksien du consommateur est calculé en utilisant un algorithme développé récemment par Breslaw et Smith (1995), qui permet également d'estimer l'écart-type associé à la valeur de la variation de surplus. Nous appliquons cette méthodologie à 31 industries agro-alimentaires correspondant à un éventail important en termes de structure concurrentielle.

Dans cet article, nous introduisons tout d'abord les concepts de base de la mesure des pertes de bien-être. Puis, nous présentons les données et les méthodes utilisées pour évaluer la marge prix-coût. Nous présentons ensuite la spécification de la demande et les procédures d'estimation. Enfin nous exposons les principaux résultats empiriques et nos conclusions.

\section{Mesure des pertes de bien-être}

C'est dans la littérature sur la taxation optimale que les problèmes de mesure des pertes de bien-être ont été le plus largement discutés (voir, entre autres, Auerbach, 1985). La première mesure proposée était basée sur la courbe de demande marshallienne. Si $x(p, y)$ représente la demande ordinaire du bien considéré, $p$ le prix du bien et $y$ le revenu du consommateur, la variation de surplus associée à un changement de prix de $p_{0}$ à $p_{1}=p_{0}+t$, où $t$ est la taxe unitaire, s'écrit

$\int_{p_{0}}^{p_{1}} x(p, y) d p$

La perte associée est définie comme la variation de surplus moins le produit de la taxe, $\left(p_{1}-p_{0}\right) x\left(p_{1}, y\right)$. Cette mesure est intuitive et facilement calculable, mais présente plusieurs désavantages. Lorsque plusieurs prix varient, sa valeur dépend de l'ordre dans lequel les prix changent. De plus, elle ne prend pas en compte la variation du niveau d'utilité entraînée par les changements de prix.

Pour mesurer la variation de bien-être, il est possible d'évaluer soit le montant que le consommateur accepterait de payer pour éviter la taxation, soit la somme que l'on devrait lui donner pour que le niveau d'utilité qu'il atteint après la taxation soit identique à son niveau d'utilité initial. Ces deux notions correspondent respectivement à la variation équivalente et à la variation compensatoire de revenu. Hicks (1942) définit la variation compensatoire de revenu, notée $C V$, comme le revenu additionnel que le consommateur doit recevoir pour que son niveau d'utilité reste constant lorsque le prix varie. Formellement, si $e(p, u)$ 
représente la fonction de dépense au niveau d'utilité $u$, alors

$$
\begin{aligned}
& C V\left(p_{0} \rightarrow p_{1}=e\left(p_{1}, v\left(p_{0}, y\right)\right)\right. \\
& \quad-e\left(p_{0}, v\left(p_{0}, y\right)\right)=e\left(p_{1}, v\left(p_{0}, y\right)\right)-y
\end{aligned}
$$

où $v(p, y)$ est la fonction d'utilité indirecte. Inversement, la variation équivalente de revenu, notée $E V$, est le revenu maximum que le consommateur serait prêt à sacrifier pour éviter le changement de prix, c'est-à-dire :

$$
\begin{aligned}
E V\left(p_{0} \rightarrow p_{1}=e\left(p_{1}, v\left(p_{1}, y\right)\right)\right. \\
\quad-e\left(p_{0}, v\left(p_{1}, y\right)\right)=y-e\left(p_{0}, v\left(p_{1}, y\right)\right)
\end{aligned}
$$

Des propriétés des fonctions de dépense, il découle que

$$
\begin{aligned}
& C V\left(p_{0} \rightarrow p_{1}\right)=\int_{p_{\mathrm{u}}}^{p_{1}} h\left(p, v\left(p_{0}, y\right)\right) d p \\
& \text { et } E V\left(p_{0} \rightarrow p_{1}\right)=\int_{p_{0}}^{p_{1}} h\left(p, v\left(p_{1}, y\right)\right) d p
\end{aligned}
$$

où $h(p, u)$ est la fonction de demande hicksienne. En particulier,

$$
C V\left(p_{0} \rightarrow p_{1}\right)=-E V\left(p_{1} \rightarrow p_{0}\right)
$$

de sorte que les mêmes méthodes peuvent être utilisées pour calculer les variations compensatoire et équivalente de revenu.

Lorsque l'utilité marginale du revenu est constante, les mesures hicksiennes sont toutes deux égales à la mesure marshallienne. Cette hypothèse est très restrictive. Cependant, Willig (1976) dérive les bornes des différences relatives entre mesures marshallienne et hicksienne, et montre que ces différences sont généralement faibles. De plus, la mesure marshallienne a l'avantage d'être basée sur la fonction de demande ordinaire qui seule est observable. Hausman (1981) montre néanmoins que les variations de surplus sous la demande hicksienne sont calculables, au moins théoriquement, à partir de la simple connaissance de la demande marshallienne. En effet, en utilisant la relation de Roy, il est possible de reconstruire la fonction d'utilité indirecte (à une constante près) ainsi que la fonction de dépense, et donc la demande hicksienne.

En pratique, la méthode proposée par Hausman est, dans bien des cas, difficile à implémenter. En effet, elle repose sur une équation différentielle qui n'a de solution analytique que dans des cas simples. Vartia (1983) propose un algorithme numérique pour calculer le revenu compensé à partir de la demande marshallienne. Breslaw et Smith (1995) améliore la méthode de Vartia en proposant un algorithme qui converge plus rapidement. Cet algorithme est basé sur une expansion de Taylor de la fonction de dépense. Si l'on considère un petit changement de prix pour un seul bien, alors

$$
\begin{aligned}
& C V\left(p_{0} \rightarrow p_{0}+\Delta p\right) \approx e\left(p_{0}, u_{0}\right) \\
& +\frac{\partial e\left(p_{0}, u_{0}\right)}{\partial p} \Delta p+0,5 \frac{\partial^{2} e\left(p_{0}, u_{0}\right)}{\partial p^{2}} \Delta^{2} p-y \\
& \approx h\left(p_{0}, u_{0}\right) \Delta p+0,5 \frac{\partial h\left(p_{0}, u_{0}\right)}{\partial p} \Delta^{2} p
\end{aligned}
$$

où $u_{0}=v\left(p_{0}, y\right)$. Pour un changement de prix de $p_{0}$ à $p_{1}$, l'intervalle est alors divisé en un grand nombre de petits intervalles, et le revenu compensé est calculé à chaque étape par la formule ci-dessus. Ceci est possible car les demandes marshallienne et hicksienne se coupent à chaque étape de l'algorithme. Ainsi, pour passer de $p_{n}$ à $p_{n+1}$, nous utilisons l'égalité

$$
\begin{aligned}
h\left(p_{n}, u_{0}\right) & =x\left(p_{n}, e\left(p_{n}, u_{0}\right)\right) \\
& =x\left(p_{n}, y+C V\left(p_{0} \rightarrow p_{n}\right)\right)
\end{aligned}
$$

Cet algorithme simple est valide pour n'importe quelle spécification de la fonction de demande marshallienne et se généralise au cas de variations simultanées de plusieurs prix. De plus, lorsque les paramètres de la fonction de demande sont estimés économétriquement, Breslaw et Smith montrent que l'on peut aisément estimer la variance de l'estimation de la variation compensatoire de revenu.

À partir des variations compensatoire et équivalente de revenu, diverses notions de pertes de bien-être peuvent être proposées. Dans le problème classique de la taxation, Mohring (1971) propose de considérer la différence entre la variation équivalente de revenu et le produit de la taxe

$$
\begin{aligned}
\operatorname{DWL}\left(p_{0} \rightarrow p_{1}\right) & =E V\left(p_{0} \rightarrow p_{1}\right) \\
& -\left(p_{1}-p_{0}\right) x\left(p_{1}, y\right)
\end{aligned}
$$

Cette quantité mesure une perte dans le sens suivant : si l'outil de collecte utilisé était un transfert forfaitaire, il serait possible de collecter jusqu'à $E V\left(p_{0} \rightarrow p_{1}\right)$ et d'amener ainsi le consommateur au même niveau d'utilité que par une taxe de $p_{0}$ à $p_{1}$. Lorsque les biens ne sont pas taxés mais subventionnés, les définitions des pertes de bien-être (qui sont alors des gains) restent valides. Cependant, diverses difficultés surgissent lorsque l'on considère des situations plus complexes. Par exemple, lorsqu'une taxe préexiste dans la situation de référence, les mesures de pertes de bien-être doivent être modifiées pour prendre en compte les effets de cette taxe initiale (Auerbach, 1985). On montre en particulier que les pertes sociales définies précédemment ne sont pas additives. Cette caractéristique constitue une limitation des mesures des pertes de bien-être. 


\section{Description des données et évaluation de l'indice de Lerner}

Le calcul de la variation de surplus du consommateur et des pertes de bien-être pour chaque branche de l'agro-alimentaire français nécessite, d'une part, l'estimation de l'équation de demande ordinaire pour chacun des produits et, d'autre part, une mesure de la marge prix-coût existant dans l'industrie permettant de calculer un prix théorique de concurrence. Au cours de cette analyse, nous utilisons deux sources principales de données :

- les données annuelles sur la consommation des ménages tirées des Comptes de la Nation, en francs courants et en francs constants de 1980, sur la période 1970-1993 (voir Insee, 1994) ;

- les données comptables issues de l'enquête annuelle menée par le Service Central des Études Économiques et Statistiques sur les entreprises de l'agro-alimentaire pour l'année 1987 (voir SCEES, 1989).

La première source de données nous permet de construire des indices implicites de prix et quantités pour chacun des produits alimentaires. L'indice de prix est obtenu en divisant les dépenses courantes par les dépenses à prix constant et constitue donc un indice de Paasche. L'indice de quantité est obtenu en divisant les dépenses à prix constants par les dépenses de l'année 1980 et constitue donc un indice de Laspeyres. De plus, les dépenses et les revenus annuels par habitant peuvent être facilement calculés en utilisant les séries des comptes nationaux de l'Insee.

Les données comptables fournies par la deuxième base de données, permettent de calculer une marge prix-coût pour les différents secteurs de l'agro-alimentaire du même type que celle calculée par le Bureau of Census américain. La marge prix-coût, notée $A P C M$, est égale à la valeur ajoutée d'une branche ( $V A$ ) diminuée des salaires $(S A)$, le tout divisé par la valeur des ventes ( $T V$ ), c'est-à-dire

$A P C M=\frac{V A-S A}{T V}$

Si $P$ représente le prix du bien, la marge prix-coût définie précédemment peut-être interprétée comme l'équivalent comptable de la mesure de l'indice de Lerner dans le cas où le coût marginal $M C$ est supposé constant et égal au coût moyen variable $A V C$ (voir Liebowitz, 1982), soit

$L \equiv \frac{P-M C}{P}=\frac{P-A V C}{P}$
La marge prix-coût peut également être déduite d'un modèle explicite d'oligopole. Par exemple, dans un jeu à la Cournot, l'indice de Lerner HPCM est donné par

$H P C M=-\frac{H H I}{\eta}$

où $\eta$ représente l'élasticité prix de la demande et $H H I$ est l'indice de concentration de Herfindahl et Hirschman (voir Cowling et Waterson, 1976). Cet indice est égal à la somme des carrés des parts de marché de toutes les firmes présentes dans l'industrie. Si toutes les firmes sont de taille identique, alors l'inverse de l'indice $H H I$ peut s'interpréter comme le nombre de firmes opérant sur le marché. Dans notre analyse, nous utilisons la relation définissant $H P C M$ pour calculer la marge prix-coût. Les calculs sont réalisés à partir des valeurs des indices $H H I$ en 1987 pour les différentes branches de l'agro-alimentaire français, tirées de Galliano (1995), et des élasticités de demande que nous avons estimées (voir ci-dessous).

En définitive, nous disposons de deux jeux de données quant aux marges prix-coût pour les 38 industries répertoriées au niveau 600 de la Nomenclature d'Activités et de Produits (NAP). Parmi celles-ci, nous avons éliminé sept branches d'activité qui soit sont des industries de transformation intermédiaires, soit sont trop diversifiées pour correspondre à un bien de la nomenclature par produits du côté consommation. Il s'agit de la semoulerie (3903), la production de malt (3906), la production de produits amylacés (3907), la production d'aliments pour animaux (3908), la fabrication d'huiles et corps gras bruts (4011), la fabrication de produits alimentaires divers (4037) et la distillation d'alcool (4101). Aussi, dans la suite, nous ne considérons que les 31 industries dont la liste est fournie dans le tableau 1 avec les indices de concentration $C 4, C 10$ et $1 / H H I$ correspondants. Les indices $C 4$ et $C 10$ représentent le pourcentage du chiffre d'affaires réalisé respectivement par les 4 et 10 premiers groupes ou entreprises indépendantes de la branche et tiennent compte des liaisons financières entre les firmes (voir Galliano, 1995).

Les mesures de marge prix-coût utilisées ne prennent pas explicitement en compte les échanges. Elles sont néanmoins valides sous certaines hypothèses. L'impact des exportations sur la mesure basée sur les données comptables est nul si les firmes réalisent des marges identiques sur les marchés intérieur et extérieur. De même, leur impact est nul sur la mesure $H P C M$ si toutes les firmes de la branche exportent leur production dans des proportions identiques. Les importations n'ont aucune incidence sur la mesure $A P C M$, mais entraînent une surestimation de la mesure basée sur l'indice de concentration puisque les firmes 
importatrices n'y sont pas comptabilisées. Le tableau 1 donne la part des exportations dans la production de chaque branche ainsi que la part des importations dans la consommation intérieure. Dans l'ensemble, la part des importations agroalimentaires est limitée dans la plupart des branches, à l'exception du riz (3905), des eaux-de-vie (4102), des conserveries de poissons (3703) et de fruits (3701) ainsi que des jus de fruits (4108). De même, les exportations ne dépassent le quart de la production que pour les eaux-de-vie (4102), le riz (3905), le champagne (4105) et le cidre (4107).

\section{Spécification et estimation de la demande marshallienne}

À l'instar de Pagoulatos et Sorensen (1986), nous utilisons la même spécification de la fonction de demande et la même procédure d'estimation pour chacune des 31 industries agro-alimentaires. Ce choix est justifié par un souci de comparabilité des résultats. La spécification choisie de la fonction de demande est de type log et est définie par

$$
\ln x_{t}^{i}=\beta_{1}+\beta_{2} \ln p_{t}^{i}+\beta_{3} \ln y_{t}+\varepsilon_{t}^{i}
$$

où $x_{t}^{i}$ est un indice de consommation par habitant du bien $i$ à la date $t, p_{t}^{i}$ est l'indice de prix du même bien déflaté par l'indice de prix à la consommation

Tableau 1 : caractéristiques de l'agro-alimentaire français en 1987

\begin{tabular}{|c|c|c|c|c|c|c|}
\hline NAP & Libellé & $C 4$ & $C 10$ & $H H I$ & Import & Export \\
\hline 3501 & Abattage du bétail & 24,3 & 37,00 & 274,7 & 22,0 & 12,7 \\
\hline 3504 & Conserve de viande & 18,8 & 28,2 & 148,8 & 7,5 & 5,2 \\
\hline 3505 & Abattage de volaille & 27,6 & 41,00 & 265,2 & 7,9 & 17,7 \\
\hline 3610 & Industrie laitière & 21,9 & 40,1 & 223,7 & 5,3 & 15,5 \\
\hline 3620 & Glaces, sorbets & 80,0 & 92,8 & 2325,6 & 8,1 & 2,2 \\
\hline 3701 & Conserverie de fruits, confiture & 44,4 & 71,1 & 787,4 & 46,7 & 21,7 \\
\hline 3702 & Conserverie de légumes & 34,4 & 51,5 & 574,7 & 21,5 & 20,1 \\
\hline 3703 & Conserverie de poissons & 32,6 & 51,7 & 392,1 & 53,5 & 16,5 \\
\hline 3704 & Plats cuisinés & 55,1 & 66,7 & 1010,1 & 2,0 & 1,7 \\
\hline 3810 & Ind. pain patisserie & 25,00 & 37,4 & 228,3 & $n c$ & $n c$ \\
\hline 3901 & Meunerie & 43,00 & 59,5 & 833,3 & 1,0 & 16,3 \\
\hline 3902 & Biscuiterie, biscotterie & 52,6 & 65,9 & 1010,1 & 25,2 & 13,6 \\
\hline 3904 & Pâtes, couscous & 84,9 & 95,7 & 3125,0 & 15,5 & 3,9 \\
\hline 3905 & Transf.céréales secondaires & 86,6 & 99,4 & 4000,0 & 81,6 & 61,8 \\
\hline 4012 & Huiles et corps gras raffinés & 84,8 & 96,1 & 2941,2 & $n c$ & $n c$ \\
\hline 4021 & Sucreries, raffinerie & 57,5 & 71,2 & 1204,8 & 10,2 & 24,7 \\
\hline 4031 & Chocolaterie, confiserie & 31,00 & 62,0 & 471,9 & 24,7 & 17,2 \\
\hline 4032 & Café, chicorée, pr.épicés & $53 ; 00$ & 79,5 & 892,8 & 17,5 & 10,9 \\
\hline 4033 & Condiments, vinaigres, sauces & 68,3 & 83,2 & 2702,7 & 32,1 & 16,9 \\
\hline 4034 & Aliments pour bébés, régime & 70,8 & 92,6 & 1960,7 & 8,6 & 24,4 \\
\hline 4035 & Entremets, petits déjeuners & 82,7 & 97,8 & 2500,0 & $n c$ & $n c$ \\
\hline 4036 & Soupes & 94,7 & 99,9 & 2857,1 & 5,2 & 5,7 \\
\hline 4102 & Eau-de-vie naturelle & 66,7 & 85,8 & 1282,0 & 68,3 & 90,8 \\
\hline 4103 & Liqueurs et apéritifs & 74,5 & 89,1 & 2777,8 & 1,7 & 18,7 \\
\hline 4104 & Apéritifs à base de vin & 97,3 & 99,7 & 4545,4 & 4,0 & 17,7 \\
\hline 4105 & Champagnisation & 52,5 & 71,3 & 1190,4 & 0,6 & 53,4 \\
\hline 4106 & Brasserie & 92,1 & 97,6 & 3846,1 & 7,5 & 4,4 \\
\hline 4107 & Cidrerie & 63,7 & 92,6 & 1408,4 & 8,3 & 30,9 \\
\hline 4108 & Jus de fruits et légumes & 65,2 & 87,2 & 1219,5 & 40,7 & 15,2 \\
\hline 4109 & Boissons non alcoolisées & 61,8 & 78,7 & 1612,9 & 8,7 & 5,9 \\
\hline 4110 & Eaux minérales naturelles & 93,2 & 97,3 & 3333,3 & 0,5 & 20,3 \\
\hline
\end{tabular}


alimentaire totale, $y_{t}$ est un indice de revenu disponible par habitant à la date $t$, les $\beta_{j}(j=1,2,3)$ sont les coefficients à estimer et $\varepsilon_{t}^{i}$ est le terme d'erreur. Les coefficients $\beta_{2}$ et $\beta_{3}$ représentent les élasticités prix et revenu de la demande. Le calcul de la variation de surplus des consommateurs et des pertes de bien-être nécessite que les conditions usuelles de régularité soient satisfaites par la fonction de demande hicksienne. En particulier (voir Deaton et Muelbauer, 1984),

$\frac{\partial h_{t}^{i}\left(p_{t}^{i}, u\right)}{\partial p_{t}^{i}}=\frac{\partial x_{t}^{i}\left(p_{t}^{i}, y_{t}\right)}{\partial p_{t}^{i}}+x_{t}^{i} \frac{\partial x_{t}^{i}\left(p_{t}^{i}, y_{t}\right)}{\partial y_{t}} \leq 0$

Cette condition implique que les coefficients $\beta_{2}$ et $\beta_{3}$ doivent vérifier l'inégalité

$\beta_{2}+w^{i} \beta_{3} \leq 0$

où $w^{i}$ représente la part de la dépense du bien $i$. Nous contraignons les coefficients de telle sorte qu'ils satisfassent cette condition pour la première année de l'échantillon (1970). L'estimation sous contrainte de l'équation de demande est menée par une procédure de moindres carrés non linéaires. Afin d'éviter les problèmes d'autocorrélation des erreurs nous avons utilisé une procédure itérative du type
Cochrane-Orcutt (voir Maddala, 1992). En effet, les premiers résultats obtenus suggéraient une forte autocorrélation des erreurs.

Les valeurs estimées des élasticités prix et revenu pour chacun des produits étudiés sont rassemblées dans le tableau 2. Dans la plupart des cas, ces élasticités sont du signe attendu. La significativité des estimations a été établie à partir des variances des estimateurs calculées en utilisant la correction de White (1981). La majorité des élasticités sont significatives, et la plupart des élasticités ne présentant pas le bon signe ne sont pas statistiquement significatives. Les valeurs des élasticités prix sont comparables à celles présentées dans Insee (1994). Par contre, leurs valeurs estimées ne corroborent pas l'hypothèse de valeur unitaire des élasticités prix fréquemment rencontrée dans la littérature sur les pertes de bien-être. Notons que les élasticités prix pour des biens tels que le champagne (4105) ou les jus de fruits (4108) sont, en valeur absolue, plus élevées que les élasticités prix des biens de base que sont le sucre (4021) et l'huile (4012). Quant aux valeurs des élasticités revenu, elles sont généralement faibles, les biens présentant les plus fortes élasticités revenu étant les crèmes glacées (3620) et les jus de fruits (4108).

Tableau 2 : élasticités estimées

\begin{tabular}{|c|c|c|c|}
\hline NAP & Libellé & Élasticité prix & Élasticité revenu \\
\hline $\begin{array}{l}3501 \\
3504 \\
3505 \\
3610 \\
3620 \\
3701 \\
3702 \\
3703 \\
3704 \\
3810 \\
3901 \\
3902 \\
3904 \\
3905 \\
4012 \\
4021 \\
4031 \\
4032 \\
4033 \\
4034 \\
4035 \\
4036 \\
4102 \\
4103 \\
4104 \\
4105 \\
4106 \\
4107 \\
4108 \\
4109 \\
4110 \\
\end{array}$ & $\begin{array}{l}\text { Abattage du bétail } \\
\text { Conserve de viande } \\
\text { Abattage de volaille } \\
\text { Industrie laitière } \\
\text { Glaces sorbets } \\
\text { Conserverie de fruits, confiture } \\
\text { Conserverie de légumes } \\
\text { Conserverie de poissons } \\
\text { Plats cuisinés } \\
\text { Ind. pain patisserie } \\
\text { Meunerie } \\
\text { Biscuiterie, biscotterie } \\
\text { Pâtes, couscous } \\
\text { Transf.céréales secondaires } \\
\text { Huiles et corps gras raffinés } \\
\text { Sucreries, raffinerie } \\
\text { Chocolaterie, confiserie } \\
\text { Café, chicorée, pr.épicés } \\
\text { Condiments, vinaigres, sauces } \\
\text { Aliments pour bébés, régime } \\
\text { Entremets, petits déjeuners } \\
\text { Soupes } \\
\text { Eau-de-vie naturelle } \\
\text { Liqueurs et apéritifs } \\
\text { Apéritifs à base de vin } \\
\text { Champagnisation } \\
\text { Brasserie } \\
\text { Cidrerie } \\
\text { Jus de fruits et légumes } \\
\text { Boissons non alcoolisées } \\
\text { Eaux minérales naturelles }\end{array}$ & $\begin{array}{c}-0,53^{(*)} \\
-0,38^{(*)} \\
-0,29^{(*)} \\
-0,42^{(*)} \\
-0,96^{(*)} \\
-0,43^{(*)} \\
-0,87^{(*)} \\
-1,08^{(*)} \\
0,47 \\
0,003 \\
-0,12^{(*)} \\
-0,24^{(*)} \\
-0,13^{(*)} \\
-0,52^{(*)} \\
-0,11^{(*)} \\
-0,53^{(*)} \\
-0,42^{(*)} \\
-0,18^{(*)} \\
-0,71^{(*)} \\
0,18 \\
0,15 \\
-0,41 \\
-0,42^{(*)} \\
-0,36^{(*)} \\
0,40^{(*)} \\
-1,44^{(*)} \\
-0,24 \\
-0,26^{(*)} \\
-1,90^{(*)} \\
-0,77^{(*)} \\
-0,70^{(*)}\end{array}$ & $\begin{array}{c}-0,13^{(*)} \\
0,14\left(^{*}\right) \\
0,11^{(*)} \\
0,15\left(^{*}\right) \\
0,64^{(*)} \\
0,19^{(*)} \\
0,06^{(*)} \\
0,41^{(*)} \\
-0,22 \\
-0,15^{(*)} \\
0,04^{(*)} \\
0,07^{(*)} \\
\left.0,044^{*}\right) \\
0,21^{(*)} \\
0,04^{(*)} \\
-0,32^{(*)} \\
0,19^{(*)} \\
-0,02 \\
0,30^{(*)} \\
-0,06 \\
-0,10 \\
0,07 \\
0,15^{(*} \\
-0,17^{(*)} \\
-0,14^{(*)} \\
0,34^{(*)} \\
-0,05 \\
-0,07^{(*)} \\
0,72^{(*)} \\
0,26^{(*)} \\
0,16\end{array}$ \\
\hline
\end{tabular}

$(*)$ : significatif à $5 \%$ 


\section{Pertes de bien-être estimées dans l'agro-alimentaire français}

Le tableau 3 fournit les estimations de la variation de surplus des consommateurs ainsi que les pertes de bien-être en 1987 pour chacune des 31 industries agro-alimentaires lorsque la marge prix-coût est calculée à partir des données comptables. Nous calculons la variation équivalente de revenu entre le prix observé $P$ et le prix théorique de concurrence, déterminé par $P \times(1-A P C M)$, en utilisant l'algorithme de Breslaw et Smith avec 300 itérations.

La variation équivalente est inférieure (respectivement supérieure) à la variation marshallienne du surplus lorsque l'élasticité revenu est positive (respectivement négative). Leur différence est toujours inférieure au double de l'écart-type de la variation équivalente. En outre, les mesures marshalliennes et hicksiennes de la variation de surplus ne diffèrent de plus de $5 \%$ que dans trois branches : crèmes glacées (3620), champagne (4105) et jus de fruits (4108). Ces branches sont caractérisées par des valeurs élevées de l'élasticité revenu. Par contre, la différence relative entre les pertes marshallienne et hicksienne de bien-être varient entre $6 \%$ et $90 \%$. Dans les deux tiers des cas, elle est comprise entre $30 \%$ et $50 \%$. Ceci confirme le fait que la mesure marshallienne des pertes de bien-être ne constitue pas en général une bonne approximation de la mesure hicksienne des pertes de bien-être.

Les pertes de bien-être estimées sont négatives pour quatre produits : plats cuisinés (3704), aliments diététiques (4034), entremets (4035), apéritifs à base de vins (4104). Pour ces industries, l'élasticité-prix de la demande compensée est quasi-nulle pour le prix observé et devient ensuite positive pour des prix inférieurs. Il faut cependant noter que, pour trois de ces secteurs, les élasticités estimées ne sont pas significatives. Si l'on ne considère que les valeurs positives, les pertes hicksiennes varient entre moins de $0,01 \%$ et $5,4 \%$ selon l'industrie considérée et la perte totale s'élève à $0,43 \%$ des dépenses alimentaires de l'année 1987. Les plus fortes pertes de bien-être sont le fait d'industries connues pour leur niveau de concentration élevé, à savoir les

Tableau 3 : pertes estimées : PCM comptable

\begin{tabular}{|c|c|c|c|c|c|c|c|}
\hline NAP & Libellé & $A P C M$ & Marsh. & $E V$ & \multicolumn{2}{|c|}{$\sigma E V$} & $D W L E V$ \\
\hline 3501 & Abattage du bétail & 3,10 & 4,90 & 4,91 & 0,26 & 0,04 & 0,05 \\
\hline 3504 & Conserve de viande & 7,14 & 13,73 & 13,66 & 0,83 & 0,19 & 0,12 \\
\hline 3610 & Industrie laitière & 2,12 & 3,92 & 3,91 & 0,06 & 0,02 & 0,01 \\
\hline 3620 & Glaces, sorbets & 10,46 & 27,25 & 25,96 & 1,54 & 1,39 & 0,10 \\
\hline 3701 & Conserverie de fruits, confiture & 7,84 & 15,03 & 14,91 & 0,51 & 0,26 & 0,14 \\
\hline 3702 & Conserverie de légumes & 8,44 & 15,74 & 15,70 & 0,52 & 0,58 & 0,54 \\
\hline 3703 & Conserverie de poissons & 6,72 & 15,24 & 14,97 & 1,10 & 0,56 & 0,29 \\
\hline 3704 & Plats cuisinés & 11,15 & 19,41 & 19,64 & 5,68 & $-0,53$ & $-0,29$ \\
\hline 3810 & Ind. pain, patisserie & 8,46 & 13,92 & 14,00 & 0,84 & $-\varepsilon$ & 0,08 \\
\hline 3901 & Meunerie & 3,10 & 5,16 & 5,16 & 0,14 & 0,01 & 0,01 \\
\hline 3902 & Biscuiterie, biscotterie & 12,28 & 23,15 & 23,03 & 1,19 & 0,36 & 0,24 \\
\hline 3904 & Pâtes, couscous & 12,54 & 22,79 & 22,73 & 0,66 & 0,19 & 0,13 \\
\hline 4012 & Huiles et corps gras raffinés & 9,72 & 14,57 & 14,55 & 0,45 & 0,08 & 0,06 \\
\hline 4021 & Sucreries, raffinerie & 8,02 & 11,42 & 11,54 & 0,47 & 0,25 & 0,37 \\
\hline 4031 & Chocolaterie, confiserie & 10,36 & 20,81 & 20,58 & 0,55 & 0,46 & 0,24 \\
\hline 4032 & Café, chicorée, pr.épicés & 7,34 & 11,78 & 11,79 & 1,26 & 0,08 & 0,09 \\
\hline 4033 & Condiments, vinaigres, sauces & 10,53 & 22,83 & 22,39 & 3,35 & 0,87 & 0,43 \\
\hline 4034 & Aliments pour bébés, régime & 10,74 & 18,35 & 18,41 & 6,51 & $-0,18$ & $-0,13$ \\
\hline 4035 & Entremets, petits déjeuners & 10,51 & 16,08 & 16,15 & 3,77 & $-0,12$ & $-0,06$ \\
\hline 4036 & Soupes & 6,86 & 12,86 & 12,83 & 3,70 & 0,19 & 0,15 \\
\hline 4102 & Eau-de-vie naturelle & 17,74 & 35,67 & 35,13 & 3,68 & 1,39 & 0,84 \\
\hline 4103 & Liqueurs et apéritifs & 36,73 & 61,55 & 63,67 & 10,58 & 4,57 & 6,69 \\
\hline 4104 & Apéritifs à base de vin & 19,07 & 23,85 & 24,09 & 1,46 & $-1,00$ & $-0,76$ \\
\hline 4105 & Champagnisation & 21,07 & 51,78 & 49,16 & 4,04 & 8,04 & 5,42 \\
\hline 4106 & Brasserie & 21,70 & 39,47 & 39,71 & 2,07 & 1,13 & 1,37 \\
\hline 4107 & Cidrerie & 13,64 & 24,84 & 24,96 & 0,96 & 0,46 & 0,58 \\
\hline 4109 & Boissons non alcoolisées & 16,39 & 36,11 & 35,13 & 2,27 & 2,37 & 1,40 \\
\hline 4110 & Eaux minérales naturelles & 19,78 & 41,33 & 40,55 & 8,41 & 3,00 & 2,22 \\
\hline
\end{tabular}

Toutes les mesures, sauf le APCM, sont exprimées en pourcentage des dépenses. 
industries des liqueurs (4103), du champagne (4105), des eaux minérales (4110) et des jus de fruits (4108). Cependant une concentration importante n'est pas toujours synonyme de perte de bien-être élevée. Par exemple, les pertes associées aux industries de production des crèmes glacées (3620), de pâtes alimentaires (3904), de riz (3905), de margarine (4012) sont parmi les plus faibles. Ainsi, il n'existe pas de corrélation forte entre le niveau de concentration d'un secteur, mesuré par un indice de type $C 4, C 10$ ou $H H I$, et l'importance (en pourcentage) des pertes de bien-être induites. Les coefficients de corrélation linéaire correspondants sont respectivement $24 \%, 25 \%$ et $23 \%$, et les coefficients de corrélation de rang de Spearman sont égaux à $39 \%, 36 \%$ et $40 \%$. Ces résultats jettent un doute sur la pratique courante qui consiste à utiliser les indices de concentration comme des indicateurs de possibles pertes de bien-être.
Le tableau 4 fournit les estimations de la variation de surplus des consommateurs ainsi que les pertes de bien-être pour chacune des 31 industries alimentaires lorsque l'indice de Lerner est calculé à partir de l'indice de concentration de Herfindahl et Hirschman. Dans un certain nombre de branches, les variations de surplus ne sont pas calculables. En effet, compte tenu des valeurs de l'indice de concentration et des élasticités prix, la valeur calculée de HPCM n'est pas toujours comprise entre 0 et $1 \mathrm{comme}$ elle devrait être. Ainsi, lorsque l'élasticité prix de la demande est positive, cet indice est négatif. De même, il est supérieur à 1 lorsque l'élasticité prix est, en valeur absolue, inférieure à l'indice $H H I$. L'hypothèse de comportement à la Cournot ne semble pas donc adéquate pour les industries en question.

Les calculs réalisés dans le contexte de l'industrie agro-alimentaire française mettent en évidence la sensibilité des évaluations de pertes de bien-être aux

Tableau 4 : pertes estimées : PCM Cournot

\begin{tabular}{|c|c|c|c|c|c|c|c|}
\hline NAP & Libellé & $P C M$ & Marsh & EV & $\sigma E V$ & DWLM & $D W L H$ \\
\hline 3501 & Abattage du bétail & 5,20 & 8,27 & 8,30 & 0,43 & 0,12 & 0,14 \\
\hline 3504 & Conserve de viande & 3,89 & 7,44 & 7,42 & 0,42 & 0,06 & 0,03 \\
\hline 3505 & Abattage de volaille & 9,11 & 15,94 & 15,86 & 0,47 & 0,22 & 0,14 \\
\hline 3610 & Industrie laitière & 5,35 & 9,96 & 9,92 & 0,16 & 0,11 & 0,07 \\
\hline 3620 & Glaces sorbets & 24,35 & 68,54 & 60,56 & 3,42 . & 8,36 & 0,38 \\
\hline 3702 & Conserverie de légumes & 6,65 & 12,30 & 12,27 & 0,37 & 0,36 & 0,33 \\
\hline 3703 & Conserverie de poissons & 3,64 & 8,12 & 8,05 & 0,57 & 0,16 & 0,09 \\
\hline 3704 & Plats cuisinés & nd & nd & nd & nd & nd & nd \\
\hline 3810 & Ind. pain patisserie & nd & nd & nd & nd & $n d$ & nd \\
\hline 3901 & Meunerie & 68,15 & 120,17 & 118,22 & 7,89 & 6,73 & 4,77 \\
\hline 3902 & Biscuiterie, biscotterie & 41,93 & 82,7 & 81,07 & 7,37 & 4,84 & 3,21 \\
\hline 3905 & Transf. céréales secondaires & 76,88 & 218,03 & 177,26 & 12,83 & 58,70 & 17,93 \\
\hline 4012 & Huiles et corps gras raffinés & 257,84 & nd & nd & nd & nd & nd \\
\hline 4021 & Sucreries raffinerie & 22,67 & 33,73 & 34,84 & 3,75 & 2,16 & 3,28 \\
\hline 4031 & Chocolaterie, confiserie & 11,25 & 22,65 & 22,38 & 0,61 & 0,55 & 0,28 \\
\hline 4032 & Café, chicorée, pr.épicés & 48,31 & 81,36 & 81,75 & 5,41 & 4,35 & 4,74 \\
\hline 4033 & Condiments, vinaigres, sauces & 38,10 & 93,33 & 85,49 & 15,91 & 13,91 & 6,07 \\
\hline 4034 & Aliments pour bébés, régime & nd & nd & nd & nd & nd & nd \\
\hline 4035 & Entremets, petits déjeuners & & nd & nd & nd & nd & $n d$ \\
\hline 4036 & Soupes & 69,30 & 157,38 & 150,18 & 69,72 & 29,30 & 22,10 \\
\hline 4102 & Eau-de-vie naturelle & 30,72 & 63,07 & 62,05 & 7,79 & 4,49 & 2,67 \\
\hline 4103 & Liqueurs et apéritifs & 77,28 & 148,46 & 169,15 & 106,21 & 28,57 & 49,26 \\
\hline 4104 & Apéritifs à base de vin & nd & nd & nd & nd & nd & nd \\
\hline 4106 & Brasserie & 155,76 & nd & nd & $n d$ & nd & nd \\
\hline 4107 & Cidrerie & 53,89 & 105,38 & 108,17 & 14,90 & 9,06 & 11,85 \\
\hline 4108 & Jus de fruits et légumes & 6,42 & 16,16 & 15,65 & 1,00 & 10,99 & 0,47 \\
\hline 4109 & Boissons non alcoolisées & 20,85 & 46,88 & 45,22 & 3,11 & 3,96 & 2,30 \\
\hline 4110 & Eaux minérales naturelles & 47,48 & 113,54 & 106,87 & 25,59 & 21,54 & 14,86 \\
\hline
\end{tabular}

Toutes les mesures, sauf le HPCM, sont exprimées en pourcentage des dépenses. nd: non défini. 
hypothèses faites sur le comportement des firmes. En effet, les estimations de variations de surplus des consommateurs et de pertes de bien-être sont généralement supérieures aux estimations présentées dans le tableau 3. Ainsi, la perte de bien-être totale représente $1,04 \%$ de la dépense alimentaire des consommateurs en 1987. Cependant, les conclusions précédentes quant à la comparaison des variations marshalliennes et hicksiennes de surplus et des pertes de bien-être s'appliquent. Les estimations de pertes de bien-être regroupées dans le tableau 4 présentent une grande variabilité. Elles sont comprises entre $0,03 \%$ pour les viandes (3504) et $49,3 \%$ pour les liqueurs (4103). Les pertes de bien-être importantes sont le fait des industries les plus concentrées, à savoir les liqueurs, les soupes (4036), le riz (3905), les eaux minérales (4110) et le cidre (4107). Ce résultat doit néanmoins être nuancé. Ainsi, dans le cas de l'industrie des soupes, les élasticités prix et revenu ne sont pas significatives. De même, l'importance des échanges pour l'industrie du riz peut entraîner une surestimation des pertes de bien-être pour cette industrie.

Le classements des industries en fonction de l'importance des pertes de bien-être calculées à partir des deux évaluations de l'indice de Lerner diffèrent sensiblement. Ainsi, les deux méthodes utilisées conduisent à des pertes de bien-être estimées qui n'ont qu'une faible corrélation entre-elles. Les coefficients de corrélation linéaire, de rang de Spearman et de rang de Kendall sont respectivement de $0,58,0,33$ et 0,23 . Ainsi, les industries qui enregistrent les plus fortes pertes de bien-être ne sont pas les mêmes selon que l'on utilise l'une ou l'autre méthode de détermination de l'écart prix-coût marginal. Ceci contredit le résultat de Peterson et Connor (1995), qui concluaient sur la base de données américaines à la convergence des résultats ordinaux obtenues à partir de dix méthodes d'évaluation différentes. Ce résultat est important car il signifie que le choix de la méthode est crucial dans le repérage des secteurs responsables des plus fortes pertes de bien-être résultant de l'existence d'un pouvoir de marché.

\section{Conclusion}

Cet article constitue une première approche des pertes de bien-être dans les industries agroalimentaires françaises. Nous avons utilisé divers développements récents sur la mesure des pertes de bien-être. Ainsi, nous avons calculé la variation hicksienne de surplus des consommateurs en utilisant un algorithme numérique construit par Breslaw et Smith (1995). De plus, à partir de nos propres estimations des demandes de biens alimentaires et de deux mesures de l'indice de Lerner, nous avons comparé les surplus et pertes de bien-être marshalliens et hicksiens. Trois conclusions peuvent être tirées de cette étude. Tout d'abord, nous montrons que si la variation de surplus marshallienne est proche de la variation équivalente du revenu, les pertes de bien-être correspondantes peuvent différer largement. Dans la mesure où l'estimation des pertes hicksiennes de bien-être peut être facilement réalisée par des algorithmes numériques, l'intérêt du recours à l'approximation marshallienne se trouve fortement réduit. Ensuite, les indices de concentration usuels ne sont pas obligatoirement de bons indicateurs de l'amplitude des pertes sociales dues au pouvoir de marché. Enfin, les méthodes de mesure de l'indice de Lerner et les hypothèses sur le comportement des firmes affectent non seulement le niveau absolu des pertes de bien-être induites par le pouvoir de marché mais aussi le classement ordinal de ces pertes ce qui milite pour une étude approfondie des différents secteurs.

Des estimations plus fiables nécessiteraient une analyse détaillée des différentes industries. En effet, si l'hypothèse d'homogénéité des biens produits peut se justifier pour certaines industries, comme les eaux minérales, elle est moins justifiée pour d'autres telles que l'industrie laitière. Cette hypothèse provient de la méthode de construction des NAP qui est basée sur une logique de substituabilité de l'offre et non de la demande. Une amélioration de l'approximation comptable de l'indice de Lerner est aussi envisageable par la prise en compte du coût du capital et d'une éventuelle appropriation de la rente due au pouvoir de marché par les salariés. Cependant, cette approximation repose implicitement sur des hypothèses technologiques (constance du coût marginal) et des hypothèses de comportement qu'il conviendrait de tester. Cette dernière piste, plus prometteuse, nécessiterait l'utilisation de données désagrégées au niveau des firmes. Il serait alors envisageable d'étudier les industries qui produisent exclusivement des biens intermédiaires, comme la malterie qui vend sa production à l'industrie brassicole. De plus, l'hypothèse d'absence de pouvoir de marché du secteur de la distribution pourrait être levée. De même, une telle approche permettrait de prendre également en compte les spécificités des différents secteurs tels que l'existence d'économies d'échelle, l'existence de réglementations ou de liens spécifiques avec l'amont ou l'aval. 


\section{Bibliographie}

Auerbach A.J. (1985). "The Theory of Excess Burden and Optimal Taxation", in Handbook of Public Economics, édité par A.J. Auerbach and M. Feldstein, North-Holland, vol. 1, pp. 61-127.

Breslaw J.A. et Smith J.B. (1995). "A Simple and Efficient Method for Estimating the Magnitude and Precision of Welfare Changes", Journal of Applied Econometrics, vol.10, pp. 313-327.

Connor J.M. et Peterson E.B. (1992). "Market-Structure Determinants of National Brand-Private Label Price Differences of Manufactured Food Products", Journal of Industrial Economics, vol. 40, pp. 157-172.

Connor J.M. et Peterson E.B. (1994). "Nouvelles estimations des pertes de bien-être et du surplus du consommateur dans l'industrie agro-alimentaire américaine", Cahiers d'Économie et de Sociologie Rurales, $\mathrm{n}^{\circ}$ 32, pp. 73-97.

Cowling K. et Waterson M. (1976). "Price-Cost Margins and Market Structure", Economica, vol. 43, pp. 267-274.

Deaton A. et Muelbauer J. (1984). Economics and Consumer Behavior, Cambridge University Press.

Galliano D. (1995). Les groupes industriels de l'agro-alimentaire français, Économica.

Gisser M. (1986). "Price Leadership and Welfare Losses in U.S. Manufacturing", American Economic Review, vol. 76, pp. 756-767.

Harberger A. (1954). "Monopoly and Resource Allocation", American Economic Review, vol. 44, pp. 77-87.

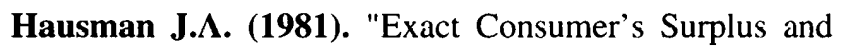
Deadweight Loss", American Economic Review, vol. 71, pp. 662-676.

Hicks J.R. (1942). "Consumer's Surplus and Index Numbers", Review of Economic Studies, vol. 9, pp. 126-137.

Insee (1994). La consommation des ménages en 1993, Série Consommation-Modes de vie, $\mathrm{n}^{\circ}$ 60-61, Ministère de l'Économie et des Finances.

Jacquemin A., Buigues A. et Ilzkovitz F. (1989). "Concentration horizontale, fusions et politique de la concurrence dans la communauté européenne", Économie Européenne, vol. 40.

Jenny F. et Weber A.P. (1983). "Aggregate Welfare Loss due to Monopoly Power in the French Economy", Journal of Industrial Economics, vol. 32, pp. 113-130.

Liebowitz S.J. (1982). "What Do Census Price-Cost Margins Measure?", Journal of Law and Economics, vol. 35, pp. 231-246.

Maddala G.S. (1992). Introduction to Econometrics, deuxième édition, Mcmillan.

Mohring H. (1971). "Alternative Welfare Gain and Loss Measures", Western Economic Journal, vol. 9, pp. 349-368.

Pagoulatos E. et Sorensen R (1986). "What Determines the Elasticity of Industry Demand?", International Journal of Industrial Organization, vol. 4, pp. 237-250.

Parker R.C. et Connor J.M. (1979). "Estimates of Consumer Loss due to Monopoly in the U.S.
Food-Manufacturing Industries", American Journal of Agricultural Economics, vol. 61, pp. 626-639.

Peterson E.B. et Connor J.M. (1995). "A Comparison of Oligopoly Welfare Loss Estimates for U.S. Food Manufacturing", American Journal of Agricultural Economics, vol. 77, pp. 300-308.

SCEES (1989). Enquête annuelle d'entreprise 1987: Industries agricoles et alimentaires, Étude $\mathrm{n}^{\circ} 285$, Ministère de l'Agriculture.

Vartia Y.O. (1983). "Efficients Methods of Measuring Welfare Change and Compensated Income in Terms of Ordinary Demand Functions", Econometrica, vol. 51, pp. 79-98.

White H. (1981). "Consequences and Detection of Misspecified Nonlinear Regression Models", Journal of the American Statistical Association, vol. 76, pp. 419-433.

Willig R.D. (1976). "Consumer's Surplus Without Apology", American Economic Review, vol. 66, pp. 589-597.

Willig R.D. (1991). "Merger Analysis, Industrial Organization, and Merger Guidelines", Brookings Papers on Economic Activity, pp. 281-332.

Willner J. (1989). "Price Leadership and Welfare Losses in U.S. Manufacturing: Comment", American Economic Review, vol. 79, pp. 604-609. 